\title{
Advanced Cervical Cancer During Pregnancy: Case Report and Review of the Literature
}

\section{Imen Ganzoui*, Dorsaf Nouri and Mariem Balti}

Radiology Department, University of Tunis El Manar, Habib Bougatfa Hospital,

Bizerta, Tunisia

*Corresponding Author: Imen Ganzoui, Head of Radiology Department, University of Tunis El Manar, Habib Bougatfa Hospital, Bizerta, Tunisia.
Received: October 12, 2021

Published: December 07, 2021

(C) All rights are reserved by Imen Ganzoui., et al.

\section{Abstract}

A 37-year-old primigravid patient, at 20 weeks of gestation, was referred to gynecology and obstetrics department for pelvic pain and vaginal bleeding. At speculum examination, she had a cervical bleeding mass. The transvaginal ultrasound showed a complex left pelvic masses. A pelvic MRI was performed, revealing an extended cervical cancer with lymph node metastasis. The patient was diagnosed with an invasive locally advanced tumor of the cervix. After multi-disciplinary reunion, pregnancy was interrupted, and the patient had hysterectomy, adnexectomy and lymphadenectomy associated with adjuvant chemotherapy. The early follow up did not reveal any complication.

Keywords: Cervical Cancer; Pregnant Woman; Locally Advanced; Lymph Nodes; Metastases; MRI

\section{Introduction}

Cervical cancer discovered during pregnancy is a rare clinical feature and a challenging malignancy, compromising both the pregnancy outcome and the future fertility [1]. About $3 \%$ of cases of cervical cancer are diagnosed during pregnancy [2]. The diagnosis of cervical carcinoma in pregnant woman is based essentially on the inspection of the uterine cervix, colposcopy with biopsy and imaging examinations. MRI is a very useful technique at every stage in the management and the staging of cervical cancer in pregnant women [3].

\section{Case Presentation}

- A 37-year-old woman, primigravid 20 weeks of gestation with unremarkable medical, surgical or family history, presented with acute hypogastric pain with vaginal bleeding.

- $\quad$ Obstetrical check-up and fetal monitoring did not reveal any abnormalities.

- At speculum examination, she had a cervical bleeding mass, and a biopsy was done.
- Blood test showed anemia and hyperleukocytosis.

- Transvaginal ultrasound demonstrated a left pelvic complex solid mass. An MRI was then indicated for further characterization. It revealed an intrauterine gestation with a cervical locally advanced cancer. The tumor was extending beyond the cervix to the upper two thirds of the vagina and the parametrium, with pelvic and paraaortic lymph nodes involvement (Figure 1).

- The patient was discussed at the tumor committee. The decision was emptying the uterine cavity by cesarean section followed by radical hysterectomy with lymphadenectomy (Figure 2). The patient was then treated with chemotherapy.

- $\quad$ There were no postoperative complications at early follow up.

\section{Discussion}

Uterine cervical cancer is one of the most common gynecological cancers. Recently malignancy during pregnancy has increased. 

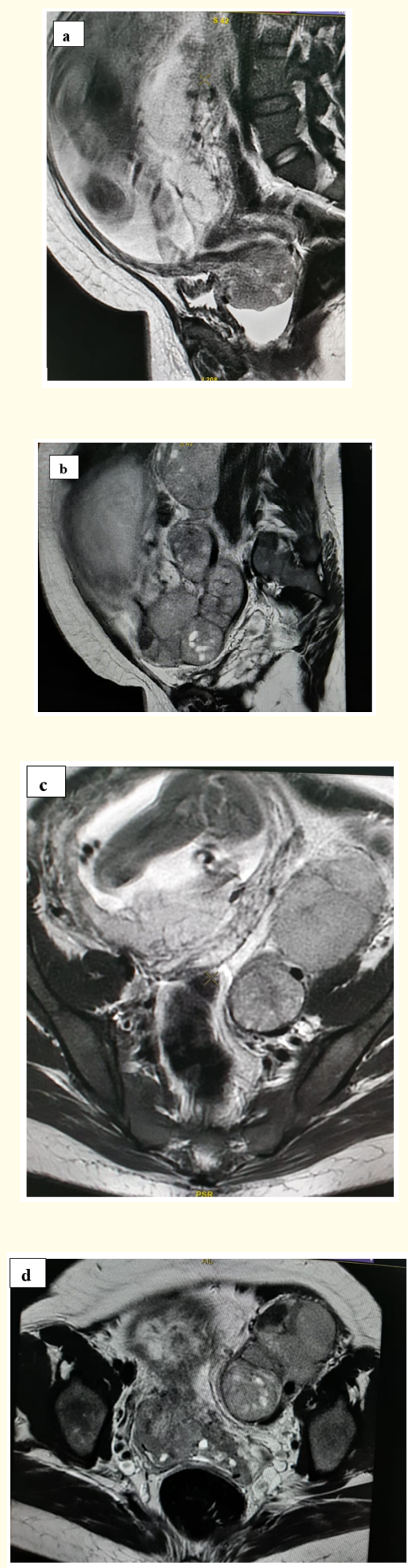

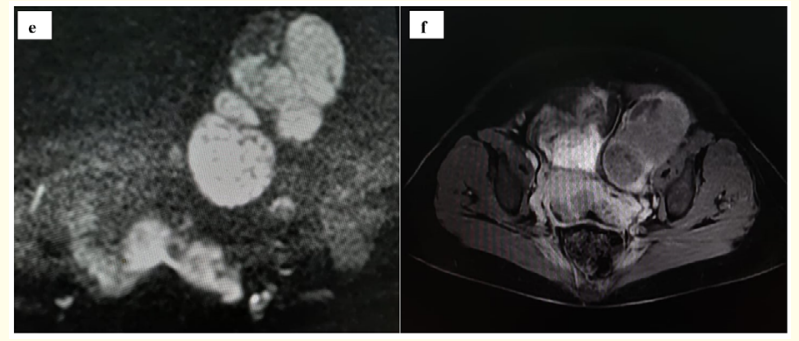

Figure 1: Sagital T2 weighted imaging $(a, b)$ and axial T2 weighted images (c, d) showing the cervical mass and the pelvic adjacent lymph nodes. Diffusion (e) and axial T1FS gadolinium weighted images showing hyperintense and heterogeneously enhanced pelvic lymph nodes.

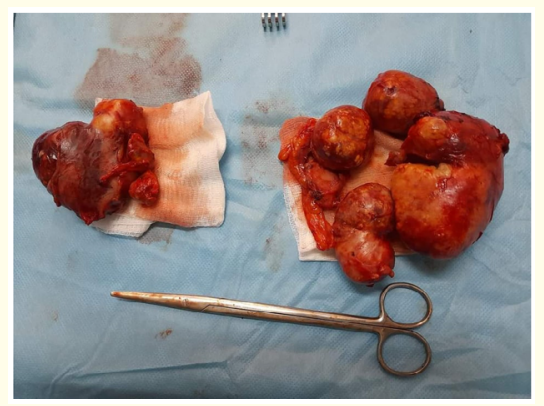

Figure 2: Per operative images after lymph nodes dissection.

Cervical cancer is the most frequent malignancy diagnosed during gestation $[4,5]$.

The mean age was 33.5 years and the mean term of pregnancy was the early second trimester [3].

Symptoms are not specific and most patients present with abnormal vaginal bleeding and abdominal pain.

Physical examination remains the first step in the management of pregnant women presenting with cervical cancer. The tumor extension may be clinically underestimated due to anatomic changes in pregnancy [1]. The cervical mass may be difficult to detect and careful examination is required to early detect the tumor. Usually, an exophytic cervical tumor is found on physical examination and biopsy is performed to confirm the malignant lesion. Evaluating 
cervical cancer propagation before surgery using pelvic MRI is indicated. The guidelines for the management of cervical cancer recommend also histological verification of suspicious lymph nodes to evaluate the cancer progression. In order to avoid an invasive procedure for pregnant patients MRI is used instead [6]. Therefore, MRI is very important for both the diagnostic and the treatment strategy in pregnant women with cervix tumor. MRI has excellent contrast enabling highly detailed assessment of loco regional tumor extent. This imaging technique has no known deleterious effects on the fetus, and its use has been advocated by the safety committee of the Society of Magnetic Resonance Imaging. Even Gadolinium Chelate Contrast agent may be administrated $t$ a pregnant woman [8]. During pregnancy the cervix may be spontaneously hyperintense, making the tumor iso or slightly hypointense on T2- weighted sequence. Diffusion-weighted imaging is also valuable for both tumor detection and lymph node extension.

There are yet some pitfulls in lesion visibility during pregnancy due to the impact of fetal movement and the volume of the uterus.

Treatment is individualized according to gestational age and cervical cancer stage [7]. Several options need to be discussed. A delay in treatment to improve the outcome of the fetus may be an option for patients with early stage cervical cancer. In patients with limited tumors, treatment may be postponed until after the delivery, especially when the diagnose was made during the middle or the end of the second trimester. A follow up with consecutive MRI is possible and conization is performed after the birth. The patients with an extended cervical tumor diagnosed during the early second trimester were treated with chemotherapy alone and the fetus was extracted after lung maturation [3]. On the other hand, some patients with extensive locally advanced cervical cancer, the gestation was medically interrupted.

\section{Conclusion}

Management of cervical cancer is more challenging during pregnancy as both maternal and fetal well-being are compromised. MRI is a mandatory examination for evaluating cervical cancer progression in pregnancy and for the decision to pursuit the pregnancy or not.

\section{Conflict of Interest}

The authors declare that there is no conflict of interest.

\section{Bibliography}

1. Zoundi Ouango 0., et al. "Uterine cervical lesion during pregnancy: diagnosis and management". Journal of Gynecology Obstetrics and Human Reproduction 3-5.3 (2006): 227-236.

2. Lishner M. "Cancer in pregnancy". Annals of Oncology S3 (2003): 31-36.

3. C Balleyguier., et al. Clinical Imaging 37 (2013) 70-76.

4. Kobayashi Y., et al. "A Japanese survey of malignant disease in pregnancy". The International Journal of Clinical Oncology 24.3 (2009): 328-333.

5. Morice P., et al. "Gynecological cancers in pregnancy". Lancet 397.9815 (2012): 558-569.

6. David Cibula., et al. "The European Society of Gynaecological Oncology/European Society of Radiotherapy and Oncology/ European Society of pathology guidelines of the management of patients with Cervical Cancer". The International Journal of Gynecological Cancer 28.4 (2018): 55-64.

7. De Haan J., et al. International Network on Cancer, Infertility Pregnancy (INCIP). "Oncological management and obstetric and neonatal outcomes for women diagnosed wih cancer during pregnancy: a 20-year international cohort study of 1170 patients". The Lancet Oncology 19 (2018): 337-346.

8. Webb JA., et al. "The use of iodinated and gadolinium contrast media during pregnancy and lactation". European Radiology 15(2015): 1234-1240.

\section{Assets from publication with us}

- Prompt Acknowledgement after receiving the article

- Thorough Double blinded peer review

- Rapid Publication

- Issue of Publication Certificate

- High visibility of your Published work

Website: www.actascientific.com/

Submit Article: www.actascientific.com/submission.php

Email us: editor@actascientific.com

Contact us: +919182824667 